\title{
Group Gap Acceptance: A New Method to Analyze Driver Behavior and Estimate the Critical Gap at Multilane Roundabouts
}

\author{
Khaled Shaaban (iD) and Hassan Hamad \\ Department of Civil Engineering, Qatar University, P.O. Box 2713, Doha, Qatar \\ Correspondence should be addressed to Khaled Shaaban; kshaaban@qu.edu.qa
}

Received 18 December 2017; Accepted 1 April 2018; Published 9 May 2018

Academic Editor: Irena I. Otković

Copyright (c) 2018 Khaled Shaaban and Hassan Hamad. This is an open access article distributed under the Creative Commons Attribution License, which permits unrestricted use, distribution, and reproduction in any medium, provided the original work is properly cited.

\begin{abstract}
The operations of multilane roundabouts, especially three-lane roundabouts, are unique and more complicated than any other type of roundabouts. This study aims to analyze driver behavior and estimate the critical gap at three-lane roundabouts. Video data were collected at two roundabouts. The analysis identified a pattern of group gap acceptance, where vehicles entering the roundabout from different lanes moved in groups during the same gap. In this case, the decision of vehicles entering from outside lanes greatly depended on the gap acceptance decision of vehicles in the inside lane. Analysis showed that the vast majority of the vehicles accept the gap in groups and the critical gap was estimated accordingly. The study provides a new explanation for the operation at multilane roundabouts. The use of this simple method is recommended when estimating critical gaps for multilane roundabouts.
\end{abstract}

\section{Introduction}

Roundabouts are popular in many countries, especially in the case of lower volumes when compared to signalized intersections as they reduce queuing and delays at the approaches. Yield-controlled entries of roundabouts oblige vehicles to decide to either reject or accept a gap in the circulating flow. The decision making in most cases depends on the driver's behavior. Roundabout capacity is greatly affected by the gap acceptance behavior of drivers. The Highway Capacity Manual (HCM) provides a method to calculate the capacity of roundabouts, and it involves a number of inputs. One of the main inputs is the critical gap [1]. This value is used in the capacity model to estimate the capacity of existing facilities without the need to perform field measurements, assess the level of service, and predict the capacity at future locations. Furthermore, this value is needed in simulation and traffic modeling of roundabouts for design and research projects.

A gap is an opening in the circulating flow that circulates around the island of the roundabout. As illustrated in Figure 1, gap vehicles are the vehicles that travel in the circulating lanes and create the gaps. The decision vehicle is the vehicle at the entry of the roundabout that takes the decision, whether rejecting or accepting a gap. The gap closes on the left side and reopens on the right side as shown in Figure 1. Rejecting a gap happens when the driver of the decision vehicle finds the gap small enough that $s$ /he refuses to merge with the circulating flow and remains stopped at the entry point. Accepting a gap is when the driver of the decision vehicle merges with the circulating flow from the vehicle's position at the entry of the roundabout as soon as the gap is large enough not to cause crashes or severe conflicts.

The critical gap is the smallest gap that a driver is assumed to accept [2]. The critical gap is an important parameter that affects the capacity and delay of roundabouts. Monitoring and evaluating of capacity values at roundabouts are a difficult matter due to the high dependence of these parameters on the drivers' behavior [1]. This study aims to investigate the gap acceptance behavior and to determine the critical gap for high-volume three-lane roundabouts. These types of roundabouts are unique, and their operation is more complicated than other types of roundabouts. Furthermore, previous studies have not thoroughly addressed these types of roundabouts to the best of our knowledge. 


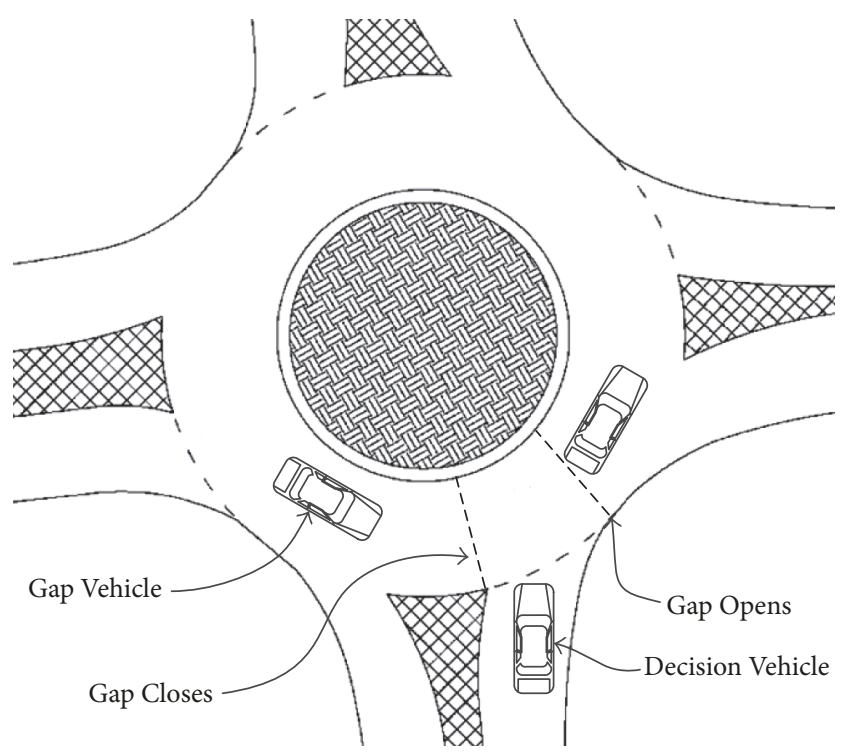

FIGURE 1: Gap acceptance definition.

\section{Literature Review}

Numerous studies have investigated critical gaps. Raff [9] developed the earliest and the most popular method of evaluating the critical lag. He defined the critical lag as the lag for which the number of accepted lags shorter than it is similar to the number of rejected lags longer than it. A graphical model was developed in which two cumulative distribution curves related to the number of accepted and rejected gaps intersect to provide the critical lag value. The original Raff's procedure estimated critical lags on the basis of lags accepted and rejected. Miller corrected the Raff's method by including the entire gap data instead of lags only [10]. This new method is known as the modified Raff method. As shown in Figure 2, the modified Raff method determines the critical gap value $\left(t_{c}\right)$, graphically, by finding the intersection point between the two functions:

$$
1-F\left(t_{r}\right), F\left(t_{a}\right)
$$

where $t_{a}$ and $t_{r}$ are accepted and rejected gap times and $F\left(t_{a}\right)$ and $F\left(t_{r}\right)$ are cumulative distribution functions (CDFs) of accepted and rejected gaps.

The intersection point between the cumulative distribution curves corresponds to the critical gap value on the horizontal axis. This value is called the critical gap because most vehicles will more likely to accept a gap of a value larger than them and reject a gap of a value smaller than them. Previous studies showed that the value of $t_{c}$ is affected by the existing traffic volumes at which this value has been evaluated $[11,12]$.

Different studies estimated the critical gap values obtained from different analysis methods and then compared them to different international standards or other studies. A summary of the critical gap values determined by previous studies is shown in Table 1 . The table contains the analysis methods, location, and types of roundabouts. In summary,

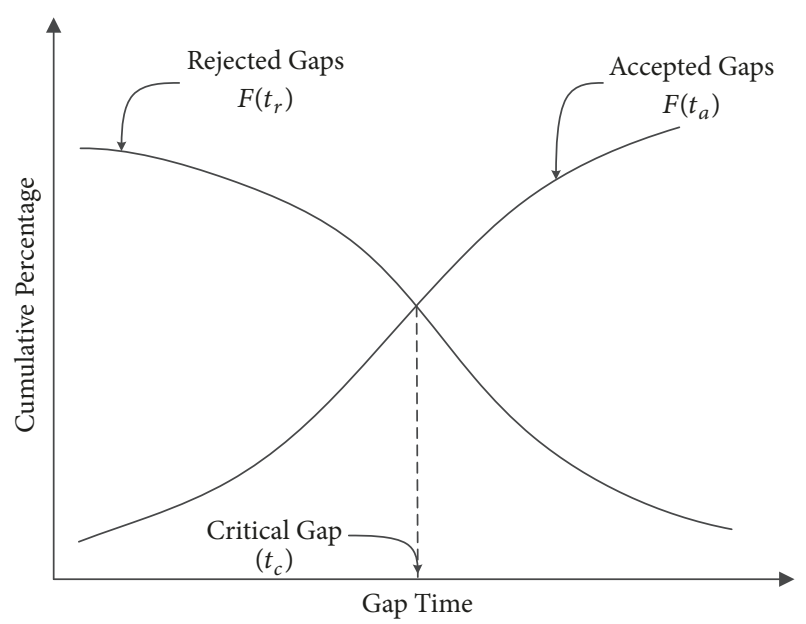

FIgURE 2: Critical gap based on Raff's method.

most studies addressed only one- and two-lane roundabouts. In summary, to our knowledge, limited studies investigated or developed a methodology to study the critical gap value for three-lane roundabouts.

Guo [7] compared three methods for the estimation of the critical gap for roundabouts, including Ashworth's method, Raff's method, and the maximum likelihood method to calculate the critical gap at roundabouts. He provided justification for recommending some of the methods over the others in practical applications. As per Guo, the modified Raff's method and the maximum likelihood method are considered easy to use and practical. On the other hand, Ashworth's method will result in a larger result because it only uses values of the accepted gap. Tupper et al. [13] compared four methods of calculating the critical gap for unsignalized T-intersections in terms of the ease of use and the use of data. Raff and the cumulative acceptance methods were identified as the most computationally simple followed closely by the fit maximization method. The equilibrium of probabilities method was the most computationally demanding. The Raff, equilibrium of probabilities, and fit maximization methods utilized both the accepted and rejected gap data, requiring a smaller sample size. The cumulative acceptance method utilized only the accepted gap data requiring a larger sample size for meaningful results. It was therefore decided to use the modified Raff's method in this study because it is easy to use, requires a small sample size, and produces reasonably accurate results.

\section{Methods}

3.1. Group Gap Acceptance. Vehicle interactions at the roundabouts vary depending on the number of circulating lanes. As the number of lanes increases, the complexity of the vehicular interactions increases. Three-lane roundabouts have complicated interaction patterns. Any two-gap vehicles in the circulating lanes regardless of the lane they are occupying can create an acceptable gap. Therefore, there should be 14 interaction cases as illustrated in Figure 3. If the decision 
TABLE 1: Summary of past studies.

\begin{tabular}{|c|c|c|c|c|c|}
\hline Author(s) & Year & Analysis method & $\begin{array}{l}\text { Critical } \\
\text { gap (s) }\end{array}$ & Type of roundabout & City, country \\
\hline Flannery and Datta [3] & 2007 & Maximum likelihood method & 3.94 & \multirow{4}{*}{ 1-lane } & Florida and Maryland, USA \\
\hline $\mathrm{Xu}$ and Tian [4] & 2008 & Maximum likelihood method & 4.8 & & California, USA \\
\hline Mensah et al. [5] & 2010 & Raff's method & 2.55 & & Maryland, USA \\
\hline Fitzpatrick et al. [6] & 2013 & Raff's method & 2.2 & & Massachusetts, USA \\
\hline $\mathrm{Xu}$ and Tian $[4]$ & 2008 & Maximum likelihood method & $\begin{array}{l}\text { L: } 4.70 / \\
\text { R: } 4.40\end{array}$ & \multirow{6}{*}{ 2-lane } & California, USA \\
\hline \multirow{4}{*}{ Guo [7] } & \multirow{4}{*}{2010} & Raff's method & 2.91 & & \multirow{4}{*}{ Dalian, China } \\
\hline & & Revised Raff's method & 2.78 & & \\
\hline & & Maximum likelihood method & 2.65 & & \\
\hline & & Ashworth method & 3.2 & & \\
\hline Kusuma and Koutsopoulos [8] & 2011 & Maximum likelihood method & 3.58 & & Stockholm, Sweden \\
\hline
\end{tabular}

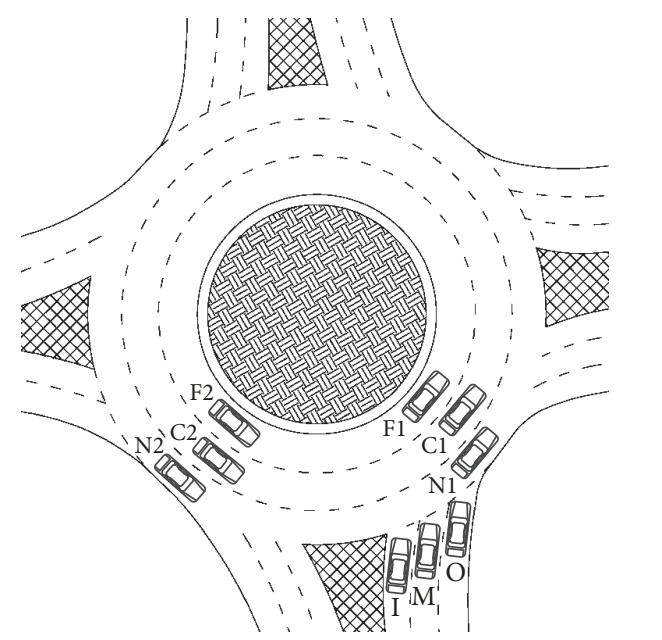

\begin{tabular}{lc}
\hline Case & Vehicle Interactions \\
\hline 1 & Vehicle I with Vehicles F1 \& F2 \\
2 & Vehicle I with Vehicles F1 \& C2 \\
3 & Vehicle I with Vehicles F1 \& N2 \\
4 & Vehicle I with Vehicles C1 \& F2 \\
5 & Vehicle I with Vehicles C1 \& C2 \\
6 & Vehicle I with Vehicles C1 \& N2 \\
7 & Vehicle I with Vehicles N1 \& F2 \\
8 & Vehicle I with Vehicles N1 \& C2 \\
9 & Vehicle I with Vehicles N1 \& N2 \\
10 & Vehicle M with Vehicles C1 \& C2 \\
11 & Vehicle M with Vehicles C1 \& N2 \\
12 & Vehicle M with Vehicles N1 \& C2 \\
13 & Vehicle M with Vehicles N1 \& N2 \\
14 & Vehicle O with Vehicles N1 \& N2 \\
\hline
\end{tabular}

$\begin{array}{ll}\text { F: Far } & \text { I: Inside } \\ \text { C: Center } & \text { M: Middle } \\ \text { N: Near } & \text { O: Outside }\end{array}$

Figure 3: Traditional interaction cases.

vehicle is in the inside lane, cases 1 through 9 are applicable covering the path of the decision vehicle and all the possible paths vehicles combinations in the circulating lanes. If the gap vehicle is in the middle lane of the approach, cases 10 through 13 are applicable. For the outside lane, only one case is applicable.

Vehicles were observed to accept gaps in groups. The group gap acceptance behavior is based on the actual mechanics of accepting or rejecting gaps observed in the field at the two study locations. Vehicles in the middle and outside lanes, follow vehicles, take advantage of the gap for the inside vehicle to enter the roundabout. As shown in Figure 4, seven cases were observed (A to G) in the field. Cases A, B, and C are based on the vehicle in the inside lane accepting a gap. Case A occurs when all lanes are occupied. In this case, the vehicle in the inside lane accepts the gap. At the same time, the follow vehicles in middle and outside lanes accept the same gap by following the vehicle in the inside lane. Case B occurs if the vehicle in the outside lane does not accept the gap with the follow vehicle in the middle lane. Case $\mathrm{C}$ occurs when the vehicle in the middle lane rejects a gap whereas the vehicles in the inside and outside lanes accept it. Case D occurs in case of no vehicles in the inside lane. In this case, the vehicle in the middle lane accepts a gap, and the follow vehicle in the outside lane accepts the same gap. The remaining cases involve only one decision vehicle. Cases E, F, and G occur if the decision vehicles accept the gap individually.

3.2. Data Collection. Video footage was used to collect the data at two high-volume three-lane roundabouts in the city of Doha, Qatar. The first roundabout is located at the intersection of Al Gharrafa Road and Al Maszhabiya Street. The second roundabout is located at the intersection of Haloul Street and Mesaimeer Road. The two selected roundabouts are similar in geometry. Both represent the typical configuration of three-lane roundabouts in the city of Doha. The circulation 

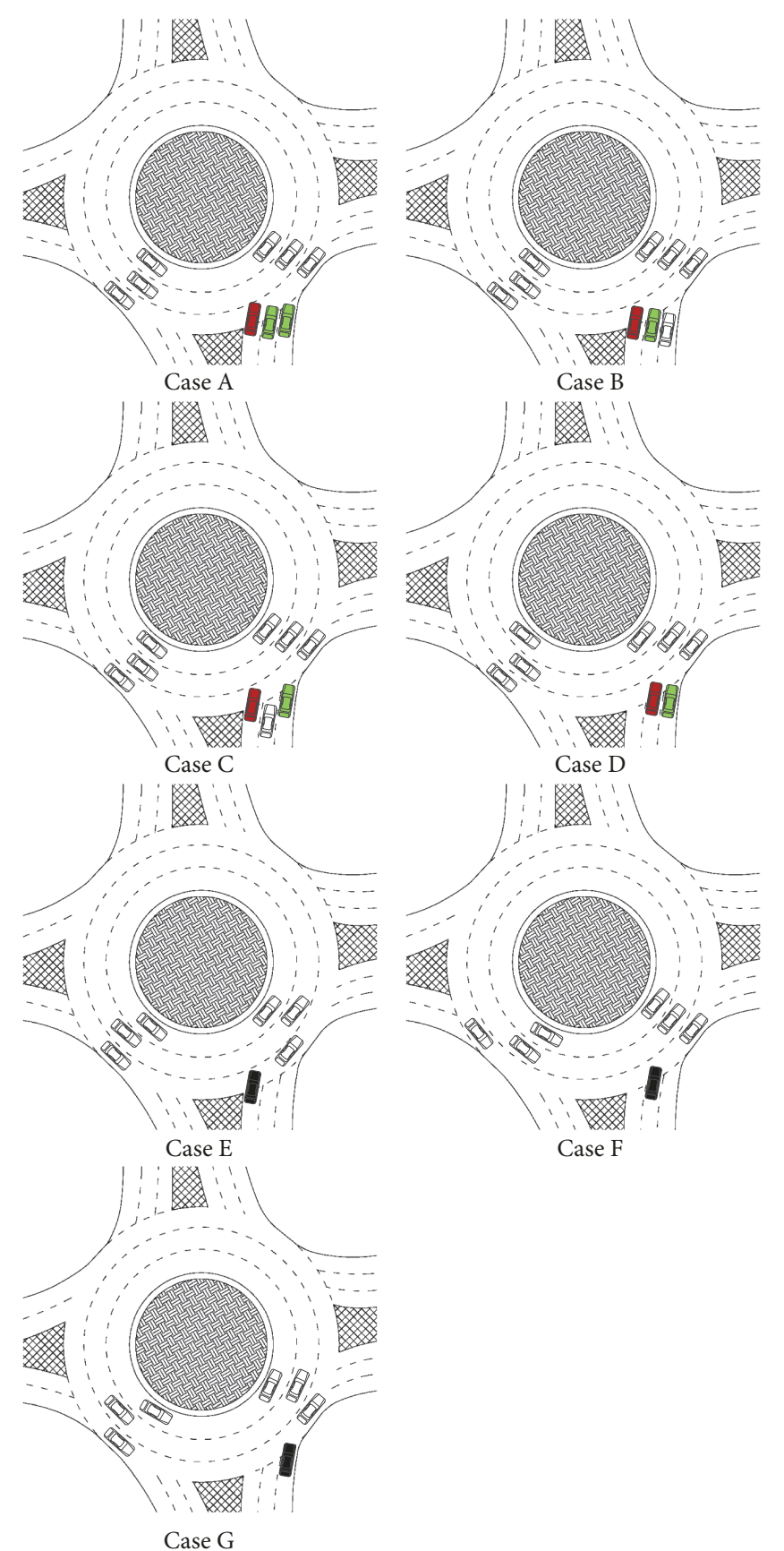

\footnotetext{
Decision vehicle accepting the gap with follow vehicles

Follow vehicle accepting the same gap of the decision vehicle

- Decision vehicle accepting the gap individually
}

FIGURE 4: Interaction cases based on group gap acceptance.

inside the roundabouts is counterclockwise in Qatar. Vehicles traveling in the circulating lanes around the middle circular island have the right-of-way, where the vehicles at the yieldcontrolled entrances have to find a gap in order to merge with the circulating flow. Both roundabouts were selected because of the heavy traffic volumes and consistent entry flow to ensure a large sample size. Cameras were installed strategically at different angles at the studied approach to ensure a clear view without the obstruction of large-sized vehicles. Data were collected for the northbound approaches at both intersections as shown in Figure 5. A total of 15 hours of footage was captured covering the peak and off-peak hours. Data collection was performed from 6:00 a.m. to 9:00 p.m. in clear weather conditions. 

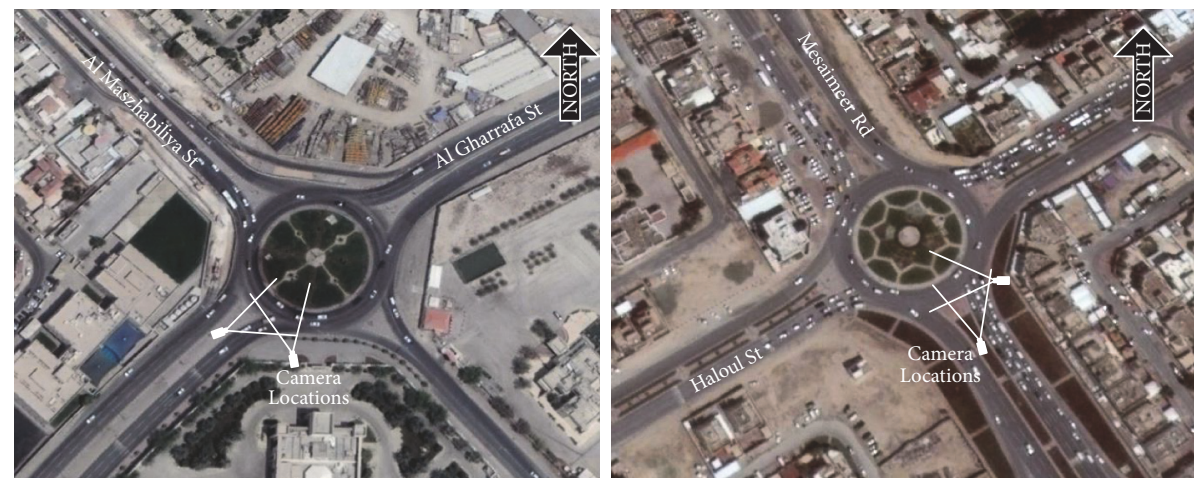

FIgURE 5: Aerial photo for roundabouts and camera locations.

3.3. Gap Measurement. The video data were analyzed frame by frame with an accuracy of $0.03 \mathrm{~s}$ based on a frame rate of 30 frames per second using Forevid analysis software to determine the time of each accepted and rejected gap. In Raff's method, the critical gap is estimated by either considering only gaps [12] or by combining gaps and lags together [14,15]. This study considered only gaps and did not consider any lags similar to Brilon et al. [15]. In this study, only decision vehicles that come to a full stop at the yield line were considered. This condition was not a problem because of the relatively large circulating volumes. The majority of the vehicles entering the roundabout stopped first before proceeding. A gap is formed in the circulating lanes by having two-gap vehicles following each other in the same or a different lane with enough gap that can fit a decision vehicle. The gap is considered accepted when the decision vehicle's driver takes the decision to proceed into a gap in the circulating flow moving from the state of full stop at the approach. On the other hand, a gap is considered rejected when the driver of the decision vehicle decides not to proceed in the case of a gap in the circulation flow regardless of its size. For the vehicles entering the roundabout, direction, vehicle type, and departure time were recorded. For the vehicles circulating in the roundabout, the passing time at specified lines across the road and direction were recorded. This information was used to identify the different interaction cases and to determine the accepted and rejected gaps for the vehicles.

3.4. Vehicle Types. In this study, vehicles were classified under three categories: passenger vehicles, medium vehicles, and heavy vehicles. Each of the used classes is basically a group of vehicles that are approximately similar in dimensions and performance. The passenger vehicles group includes sedans, sport-utility vehicles, pickup trucks, and vans. Medium vehicles group includes single-unit two-axle trucks, small recreational vehicles, minibuses, and ambulances. Heavy vehicles group includes large buses, trailers of all sizes, and dump trucks. The summary of the collected data is presented in Table 2. Most of the vehicles in the sample (95.47\%) were passenger vehicles.

3.5. Interaction Cases. The vehicular interaction cases are not similar amongst the different types of roundabouts because the number of lanes in each type of the roundabouts dictates the complexity of the interaction cases. Three-lane roundabouts have complex interaction cases, as explained earlier, and, due to that, new interaction cases were developed in a way that reflects the driver decision-taking more accurately. Some of the cases rarely occurred such that there are not enough data points to perform the analysis. Only the cases that have an abundance of data points have been analyzed. The total number of observed interactions exceeded 4,500 interactions over the duration of the data collection for both locations. As indicated in Table 2, case A was the most occurring case with $74.98 \%$ of all cases.

\section{Results}

4.1. Overall Critical Gap. Using the modified Raff's method, the overall critical gap, defined as the intersection between the CDFs of the rejected and accepted gaps at which the probability of rejecting or accepting, was estimated for all the data points. This value was estimated by equalizing the sigmodal functions of the cumulative distribution plots. The overall critical gap for the two roundabouts was 2.40 seconds. Figure 6 shows the CDFs that yielded the critical gap value of the two three-lane roundabouts.

4.2. Critical Gap for the Interaction Cases. Most of the interaction cases observed were case $\mathrm{A}$, where the vehicles at the roundabout entry proceed together. The critical gap value for case A was $2.45 \mathrm{~s}$, which is close to the overall critical gap value. A summary of the overall critical gap, the critical gaps of the different interaction cases, and vehicle types are listed in Table 3. Figure 7 shows the graphical representation of the data based on the modified Raff's definition to obtain the critical gap for each vehicular interaction case.

4.3. Critical Gap for the Vehicle Types. It was found that the critical gap values for passenger, medium, and heavy vehicles are in an ascending order. The passenger vehicles had a critical gap value of $2.39 \mathrm{~s}$ compared to $2.53 \mathrm{~s}$ and $3.03 \mathrm{~s}$ for medium and heavy vehicles, respectively. The number of data points for medium and heavy vehicles shows that more data points are required to obtain more accurate results. Figure 7 shows the graphical representation of modified Raff's method for the different vehicle types. 
TABLE 2: Summary of data collected.

\begin{tabular}{|c|c|c|c|c|c|c|c|}
\hline \multirow{2}{*}{$\begin{array}{l}\text { Location } \\
\text { Classification }\end{array}$} & \multirow[b]{2}{*}{ Type } & \multicolumn{2}{|c|}{ Al Gharrafa St. roundabout } & \multicolumn{2}{|c|}{ Haloul St. roundabout } & \multicolumn{2}{|c|}{ Combined } \\
\hline & & Count & Percentage & Count & Percentage & Count & Percentage \\
\hline \multirow{2}{*}{ Gap } & Accepted & 1025 & $44.55 \%$ & 1112 & $46.66 \%$ & 2137 & $45.62 \%$ \\
\hline & Rejected & 1276 & $55.45 \%$ & 1271 & $53.34 \%$ & 2547 & $54.38 \%$ \\
\hline \multirow{3}{*}{ Vehicle type } & Passenger vehicle & 2210 & $96.05 \%$ & 2262 & $94.92 \%$ & 4472 & $95.47 \%$ \\
\hline & Medium vehicle & 62 & $2.69 \%$ & 63 & $2.64 \%$ & 125 & $2.67 \%$ \\
\hline & Heavy vehicle & 29 & $1.26 \%$ & 58 & $2.43 \%$ & 87 & $1.86 \%$ \\
\hline \multirow{4}{*}{ Group gap } & A & 1860 & $80.83 \%$ & 1652 & $69.32 \%$ & 3512 & $74.98 \%$ \\
\hline & B & 118 & $5.13 \%$ & 302 & $12.67 \%$ & 420 & $8.97 \%$ \\
\hline & $\mathrm{C}$ & 205 & $8.91 \%$ & 180 & $7.55 \%$ & 385 & $8.22 \%$ \\
\hline & $\mathrm{D}$ & 23 & $1.00 \%$ & 4 & $0.17 \%$ & 27 & $0.58 \%$ \\
\hline \multirow{3}{*}{ Individual gap } & $\mathrm{E}$ & 87 & $3.78 \%$ & 239 & $10.03 \%$ & 326 & $6.96 \%$ \\
\hline & F & 6 & $0.26 \%$ & 5 & $0.21 \%$ & 11 & $0.23 \%$ \\
\hline & G & 2 & $0.09 \%$ & 1 & $0.04 \%$ & 3 & $0.06 \%$ \\
\hline Total & & 2301 & $100 \%$ & 2383 & $100 \%$ & 4684 & $100 \%$ \\
\hline
\end{tabular}

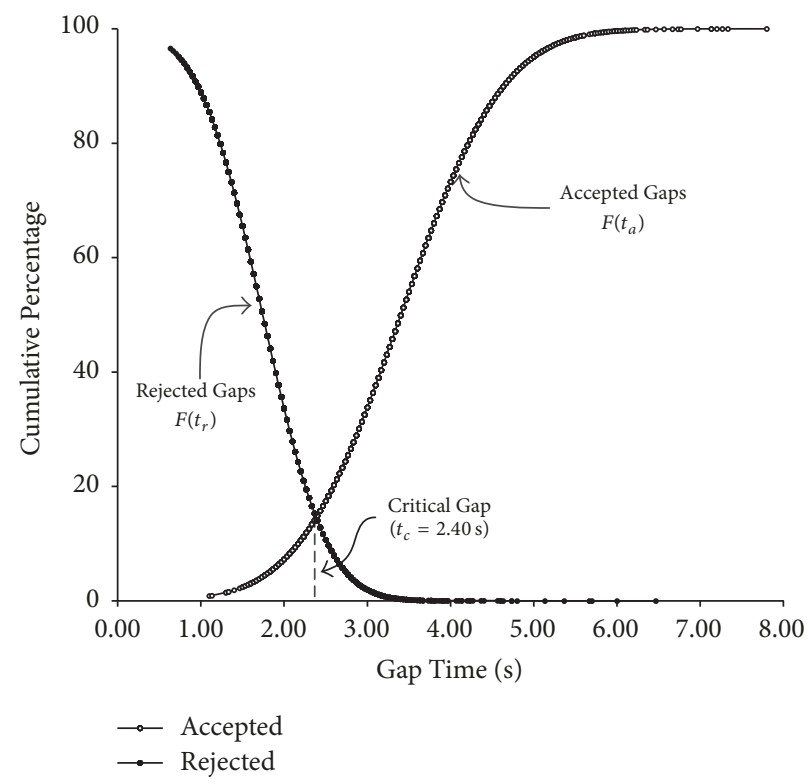

FIgURE 6: Overall critical gap.

\section{Conclusions}

The presented method, group gap acceptance, was used to analyze driver behavior and estimate the critical gap for threelane roundabouts. Data were collected at two roundabouts in the city of Doha, Qatar. More than 4,500 interactions were recorded. Data were classified based on the vehicle type and interaction between vehicles. Field results indicated that the gap acceptance decision for vehicles entering from the inside lane affects the decision of vehicles entering from the outside lanes. In this behavior, named group gap acceptance, the vehicles in the outside lanes follow the vehicle in the inside lane and enter the roundabout during the same gap.

The overall critical gap value was $2.40 \mathrm{~s}$. The critical gap for passenger vehicles was the lowest $(2.39 \mathrm{~s})$ compared to medium $(2.53 \mathrm{~s})$ and heavy vehicles $(3.03 \mathrm{~s})$. Nine driver interaction cases were identified (A-G). Most of the interactions (92.75\%) involved more than one vehicle entering the roundabout and moving together in groups. Case A, three vehicles in a group, was the dominant interaction case, accounting for more than $74.98 \%$ of the data points collected from two locations, with a critical gap value of $2.45 \mathrm{~s}$.

The presented method, group gap acceptance, was used to analyze driver behavior and estimate the critical gap for three-lane roundabouts. The critical gap for three-lane roundabouts, but the value of the overall critical gap, seems to be much lower than that obtained for one- and two-lane roundabouts [3-8]. Considering that driver behavior is a major contributor to the operational performance of roundabouts, the results may be an indication of aggressive driver behavior, which was identified in prior studies conducted 

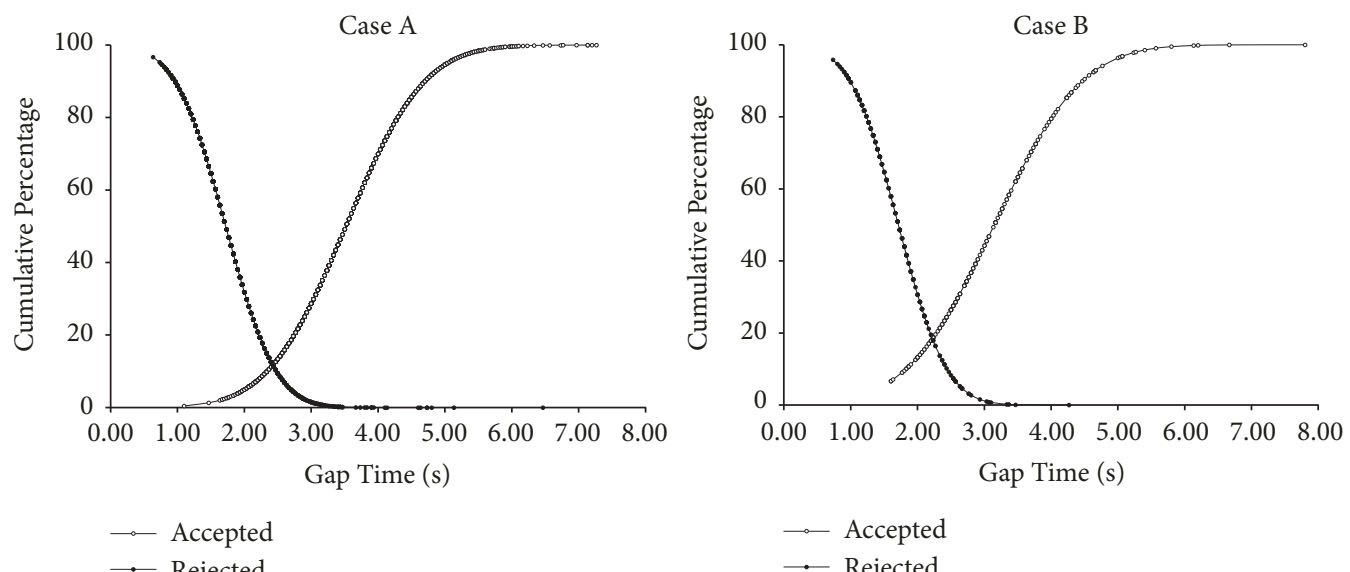

$\longrightarrow$ Accepted

$\longrightarrow$ Rejected
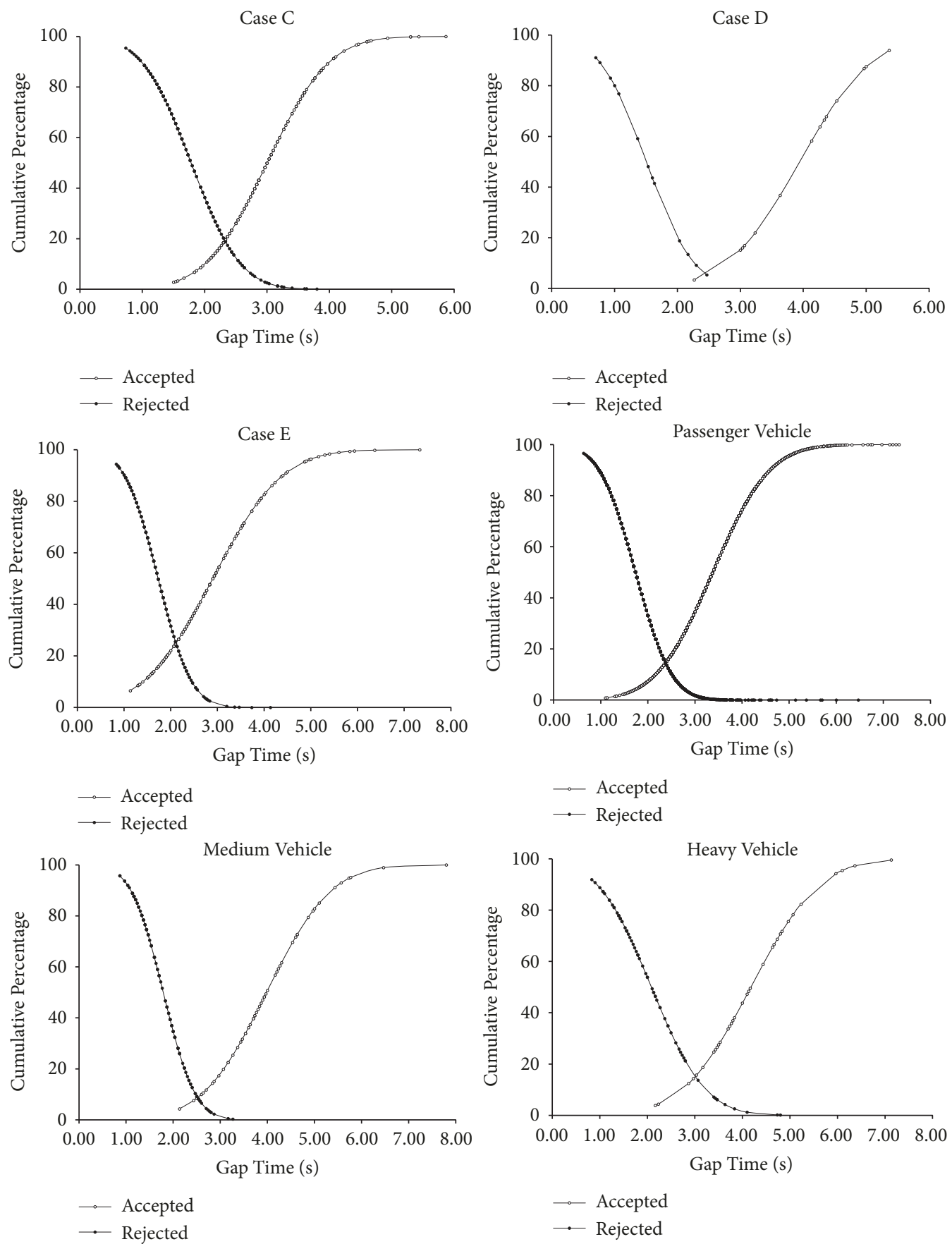

Figure 7: Critical gap for different cases. 
TABLE 3: Summary of critical gap values.

\begin{tabular}{lcc}
\hline Classification & Type & Critical gap (s) \\
\hline Overall & & 2.40 \\
\hline \multirow{3}{*}{ Vehicle types } & Passenger vehicle & 2.39 \\
& Medium vehicle & 2.53 \\
& Heavy vehicle & 3.03 \\
\hline A & 2.45 \\
Interaction cases & B & 2.22 \\
& C & 2.33 \\
& D & 2.47 \\
E & 2.11 \\
& F & Not enough data \\
& G & Not enough data \\
\hline
\end{tabular}

in Qatar [16-18]. Such behavior may be caused by drivers becoming frustrated at heavily congested roundabouts and entering the roundabout when there is an insufficient gap.

The values obtained in this study can be used as a baseline for critical gap values of three-lane roundabouts in Qatar and other countries in the region, such as Saudi Arabia, United Arab Emirates, Bahrain, Kuwait, and Oman. These values can be used in numerous applications. For example, they can be input in capacity-prediction models and used in the simulation of traffic flow at multilane roundabouts to replicate real-life conditions. The method developed in this study can also be employed to estimate critical gap values for different types of multilane roundabouts in other regions.

The current study has a number of limitations. It did not consider the effect of pedestrians or cyclists on critical gap values because no pedestrians or cyclists were observed at the study locations, as expected throughout this region [19]. Furthermore, this study did not consider the effects of the geometry of the roundabout. Some roundabouts have an elliptical shape, whereas others have a steep slope, with circulating lanes, three legs, or slip ramps. In the current study, both roundabouts were circular, flat, and had four legs. Furthermore, the approaches to the roundabout did not feature slip ramps. In addition, the vehicular interaction cases in the study were based on allowable legal movements. Driving behaviors considered illegal, such as a vehicle entering the far circulating lane from the outside lane, were not included, as such behaviors, besides being careless, high-risk maneuvers, are in violation of traffic law and do not depict acceptable gap acceptance maneuvers.

\section{Disclosure}

The statements made herein are solely the responsibility of the authors.

\section{Conflicts of Interest}

The authors declare that there are no conflicts of interest regarding the publication of this paper.

\section{Acknowledgments}

This publication was made possible by the Qatar Research Fund (a member of Qatar Foundation) through an NPRP award [NPRP 4-1170-2-456].

\section{References}

[1] C: National Research and B: Transportation Research, HCM 2010 : highway capacity manual, Transportation Research Board, Washington, Wash, USA, 2010.

[2] R. J. Troutbeck, "Estimating the mean critical gap," Transportation Research Record, vol. 2461, pp. 76-84, 2014.

[3] A. Flannery and T. Datta, "Operational performance measures of American roundabouts," Transportation Research Record, vol. 1572, pp. 68-75, 1997.

[4] F. Xu and Z. Z. Tian, "Driver behavior and gap-acceptance characteristics at roundabouts in California," Transportation Research Record, vol. 2071, no. 1, pp. 117-124, 2008.

[5] S. Mensah, S. Eshragh, and A. Faghri, "A critical gap analysis of modern roundabouts," in Proceedings of the 89th Annual Meeting of the Transportation Research Board, Washington, Wash, USA, January 2010.

[6] C. Fitzpatrick, D. Abrams, Y. Tang, and M. Knodler, "Spatial and temporal analysis of driver gap acceptance behavior at modern roundabouts," Transportation Research Record, no. 2388, pp. 1420, 2013.

[7] R. Guo, "Estimating critical gap of roundabouts by different methods," in Proceedings of the 6th Advanced Forum on Transportation of China (AFTC 2010), pp. 84-89, Beijing, China, 2010.

[8] A. Kusuma and H. N. Koutsopoulos, "Critical gap analysis of dual lane roundabouts," pp. 709-717.

[9] M. S. Raff, “A volume warrant for urban stop signs," 1950.

[10] A. J. Miller, "A note on the analysis of gap-acceptance in traffic," Journal of the Royal Statistical Society, vol. 23, no. 1, pp. 66-73, 1974.

[11] A. Miller, "Nine estimators of gap-acceptance parameters," in Bulletin Transport Section, Civil Engineering, vol. 5, 1971.

[12] W. Brilon, R. Koenig, and R. J. Troutbeck, "Useful estimation procedures for critical gaps," Transportation Research Part A: Policy and Practice, vol. 33, no. 3-4, pp. 161-186, 1999.

[13] S. Tupper, M. A. Knodler Jr, C. Fitzpatrick, and D. S. Hurwitz, "Estimating Critical Gap-A Comparison of Methodologies Using a Robust, Real-World Data Set," in Proceedings of the Transportation Research Board 92nd Annual Meeting, 5019, 13 pages, 2013.

[14] R. Ashalatha and S. Chandra, "Critical gap through clearing behavior of drivers at unsignalised intersections," KSCE Journal of Civil Engineering, vol. 15, no. 8, pp. 1427-1434, 2011.

[15] W. Brilon, R. Koenig, and R. J. Troutbeck, "Useful estimation procedures for critical gaps," Transportation Research Part A: Policy and Practice, vol. 33, no. 3, pp. 161-186, 1999.

[16] K. Shaaban, J. S. Wood, and V. V. Gayah, "Investigating driver behavior at minor-street stop-controlled intersections in Qatar," Transportation Research Record, vol. 2663, pp. 109-116, 2017.

[17] A. Tageldin, T. Sayed, and K. Shaaban, "Comparison of timeproximity and evasive action conflict measures case studies from five cities," Transportation Research Record, vol. 2661, pp. 19-29, 2017.

[18] K. Shaaban and A. Pande, "Evaluation of red-light camera enforcement using traffic violations," Journal of Traffic and 
Transportation Engineering (English Edition), vol. 5, no. 1, pp. 66-72, 2018.

[19] K. Shaaban, D. Muley, and A. Mohammed, "Analysis of illegal pedestrian crossing behavior on a major divided arterial road," Transportation Research Part F: Traffic Psychology and Behaviour, vol. 54, pp. 124-137, 2018. 


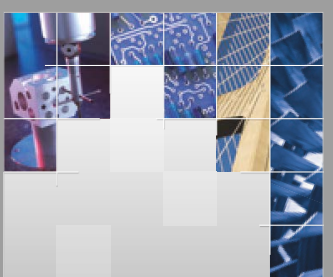

\section{Enfincering}
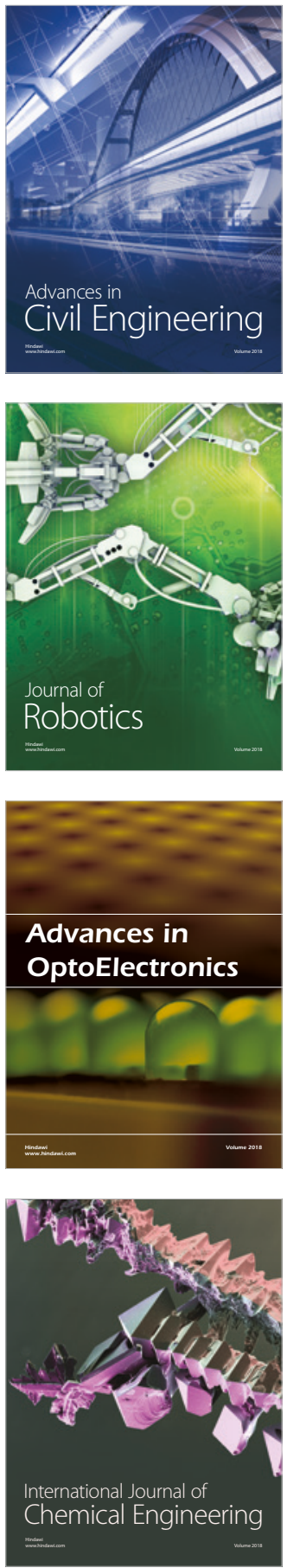

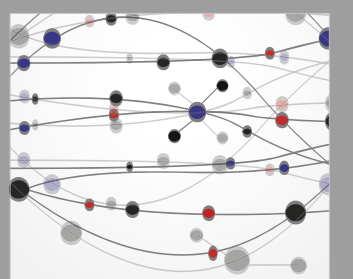

\section{Rotating \\ Machinery}

The Scientific World Journal

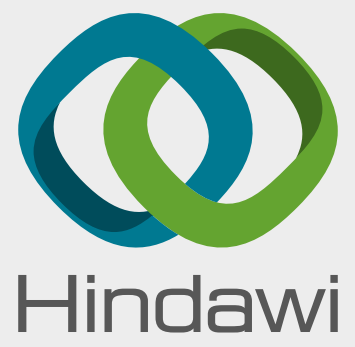

Submit your manuscripts at

www.hindawi.com
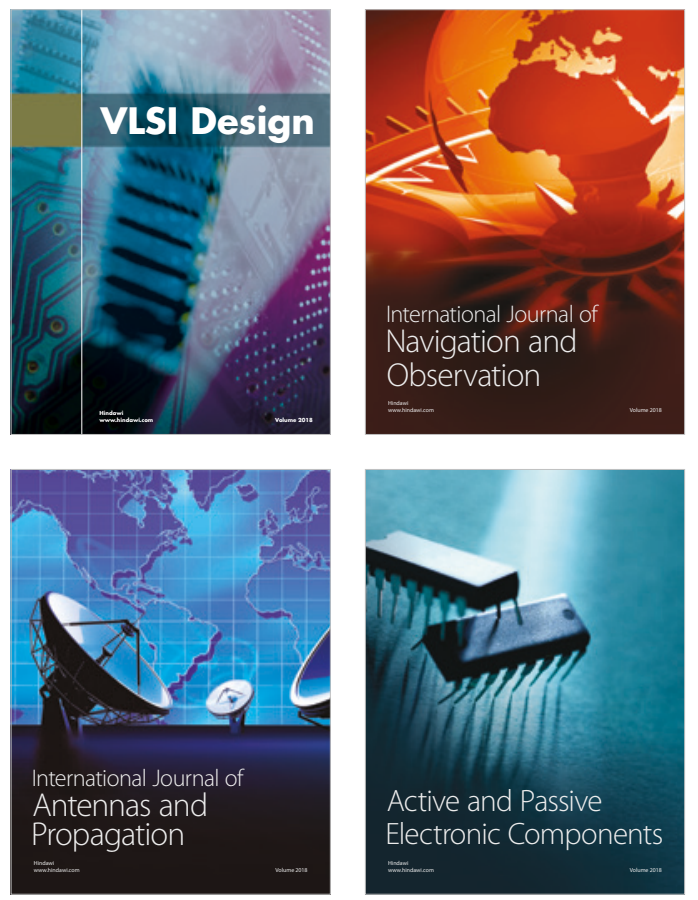
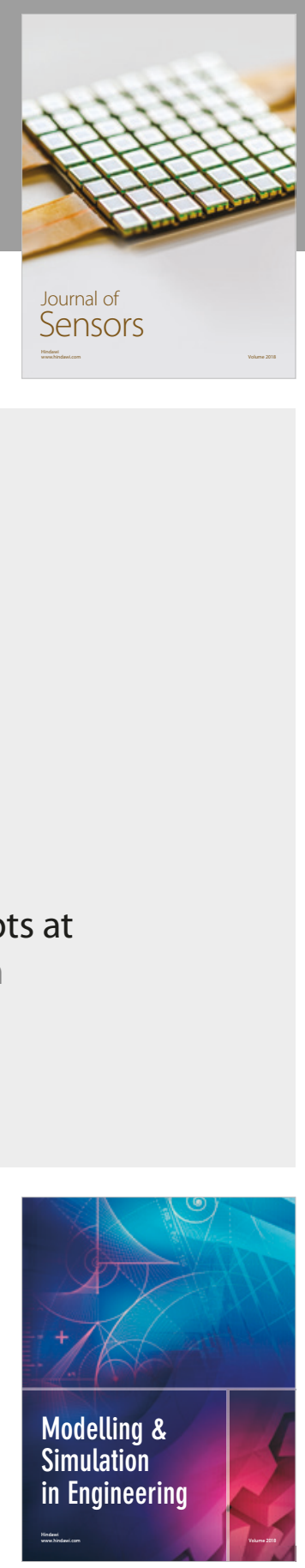

\section{Advances \\ Multimedia}
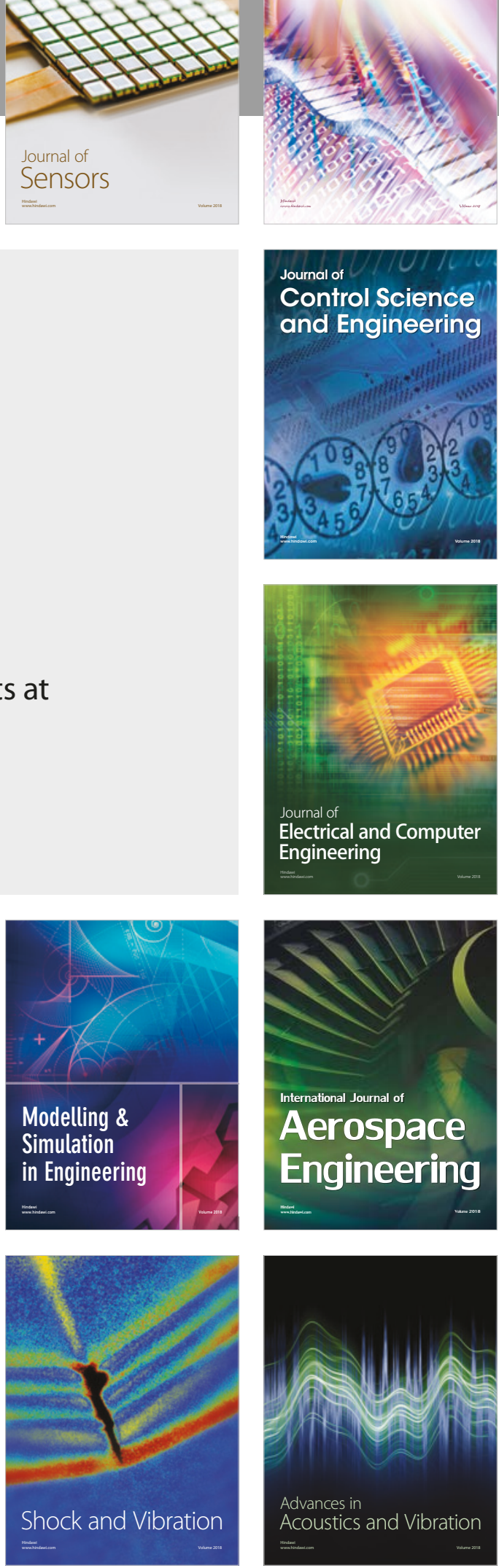